\title{
Learning Temporally Precise Spiking Patterns through Reward Modulated Spike-Timing-Dependent Plasticity
}

\author{
Brian Gardner and André Grüning \\ Department of Computing, University of Surrey, \\ Guildford, Surrey, GU2 7XH, United Kingdom \\ \{b.gardner, a.gruning\}@surrey . ac.uk
}

\begin{abstract}
Precise neuronal spike timing plays an important role in many aspects of cognitive processing. Here, we explore how a spiking neural network can learn to generate temporally precise spikes in response to a spatio-temporal pattern, through spike-timing-dependent plasticity modulated by a delayed reward signal. An escape noise neuron is implemented as the readout to incorporate the effect of background noise on spike timing. We compare the performance of two different escape rate functions that drive spiking in the readout neuron: the Arrhenius \& Current $(\mathrm{A} \& \mathrm{C})$ and Exponential (EXP) model. Our results show that the network can learn to reproduce target spike patterns containing between 1 and 10 spikes with $10 \mathrm{~ms}$ temporal accuracy. We also demonstrate the superior performance of the A\&C model over the EXP model for the parameters we consider, especially when reproducing a large number of target spikes.
\end{abstract}

Keywords: Neuronal Plasticity, Stochastic Neuron, Synapses

\section{Introduction}

Generating temporally precise sequences of spikes in response to synaptic input is a fundamental process of neural activity [3]. Learning to generate such responses is considered to take place in the brain through the modification of synaptic strengths between neurons. A variety of synaptic processes are found to drive short to longer term synaptic changes [13]. In particular, Spike-TimingDependent Plasticity (STDP) seems to play a key role in learning: a process experimentally observed in hippocampal neurons [2]. A method proposed by Ponulak et al. [15], Remote Supervised learning Method (ReSuMe), demonstrated how such an STDP process can be applied in teaching a network of spiking neurons to map input patterns to arbitrary target spike trains. Furthermore, this method was extended to learning in multilayered networks $[10,17]$. However, whilst ReSuMe retains a good degree of biological plausibility through the inclusion of STDP, it is uncertain how a feedback signal in the form of a target spike train can realistically be communicated instantaneously during learning. 
Reward modulated STDP has emerged as a more plausible hypothesis for learning with spiking neurons, where time-dependent correlations in the spiking activity drive synaptic strength modifications, subject to a global reward signal $[7,11,12]$. For learning a target spike train, this corresponds to strengthening synapses associated with triggering the correct firing times. Previous investigations have demonstrated how networks of spiking neurons learn to reproduce temporally precise sequences of spikes with reward modulation $[6,12,8,5]$. Furthermore, the importance of background noise has been indicated, where varying neuronal spiking activity is essential for the exploration of reward space towards discovering those spiking patterns that are desired.

In this paper, we implement a stochastic neuron model and investigate its ability to learn a target spike train in response to a spatio-temporal spiking pattern, through a reward-maximising STDP rule [8]. For biological plausibility, only delayed reward signals are considered, where feedback on the correctness of a response becomes available upon cessation of the input pattern. We also consider two different forms for the escape rate to drive neuronal spiking and compare their performance over a range of target spike trains.

\section{Method}

\subsection{Single Neuron Model}

We consider a two-layer feedforward neural network based on $[14,18,9]$. A single readout neuron receives input from $1 \leq j \leq M$ presynaptic neurons. The spike train received from the $j^{\text {th }}$ neuron is denoted $X_{j}$, and the spatio-temporal spiking pattern over all $M$ inputs is $\mathbf{X}=\left\{X_{1}, \ldots, X_{M}\right\}$. If the readout neuron generates the output spike train $Y$ in response to $\mathbf{X}$, then its membrane potential at time $t$ is:

$$
u(t \mid \mathbf{X}, Y):=U_{\text {rest }}+\sum_{j=1}^{M} w_{j} \sum_{s \in X_{j}} \epsilon(t-s)+\sum_{s \in Y} \kappa(t-s)
$$

with $U_{\text {rest }}=-70 \mathrm{mV}$ the resting membrane potential and $w_{j}$ the $j^{\text {th }}$ afferent synaptic weight. We approximate the Postsynaptic Potential (PSP) kernel as a double exponential: $\epsilon(s)=\epsilon_{0}\left(\mathrm{e}^{-s / \tau_{m}}-\mathrm{e}^{-s / \tau_{s}}\right)$, and the reset kernel: $\kappa(s)=$ $\kappa_{0} \mathrm{e}^{-s / \tau_{m}}$; both kernels are set to 0 for $s<0$. We set $\epsilon_{0}=1.3 \mathrm{mV}$, such that a synaptic weight $w_{j}=1$ evokes a PSP with an absolute amplitude close to 1 $\mathrm{mV}$, and we set $\kappa_{0}=-10 \mathrm{mV}$. The membrane time constant is set to $\tau_{m}=10$ $\mathrm{ms}$ and the synaptic time constant $\tau_{s}=0.7 \mathrm{~ms}$.

To account for background noise, we implement an escape noise model [9]. Spiking events are driven by an escape rate $\rho(u(t))$, that gives the instantaneous firing density for the readout neuron as a function of the time-dependent membrane potential. We set the simulation time step $\delta t=1 \mathrm{~ms}$. 
In our simulations we consider two different functional forms for the escape rate, the first being the Arrhenius \& Current (A\&C) [9]:

$$
\rho^{\mathrm{A} \& \mathrm{C}}(u, \dot{u})=2\left(\frac{c_{1}}{\tau_{m}}+\frac{c_{2}}{\sigma}[\dot{u}]_{+}\right) \frac{\exp \left\{-\frac{[u-\vartheta]^{2}}{\sigma^{2}}\right\}}{1+\operatorname{erf}\left\{-\frac{u-\vartheta}{\sigma}\right\}},
$$

with the firing threshold set to $\vartheta=-55 \mathrm{mV}$. The parameter $\sigma$ is the noise amplitude, corresponding to the magnitude in the fluctuations of $u$ due to background stochastic spike arrival. We set $\sigma=5 \mathrm{mV}$, mimicking that measured from in vivo experiments [4]. The parameters $c_{1}$ and $c_{2}$ are set to 0.72 and $\frac{1}{\sqrt{\pi}}$ respectively [9]. The term $[\dot{u}]_{+}$indicates that only positive gradients in the membrane potential contribute to the firing density. The error function erf ensures a linear increase in the firing density for $u>\vartheta$.

The second simpler model, referred to as Exponential (EXP), is more commonly used [9]:

$$
\rho^{\mathrm{EXP}}(u)=k \exp \{\beta(u-\vartheta)\} .
$$

We set the stochasticity parameters $k=0.156$ and $\beta=0.334$, such that for $u<\vartheta: \rho^{\operatorname{EXP}}(u) \approx \rho^{\mathrm{A} \& \mathrm{C}}(u, \dot{u}=0)$, giving comparable levels of noise between the two models.

\subsection{Learning Algorithm}

A stochastic neuron model allows for the determination of the likelihood for generating the set of output spikes $Y$ in response to $\mathbf{X}$. By the technique of gradient ascent, the eligibility for the $j^{\text {th }}$ synapse can be found as [14,7]:

$$
e_{j}(t)=\frac{\rho^{\prime}(u)}{\rho(u)}[\mathcal{Y}(t)-\rho(u)] \sum_{s \in X_{j}} \epsilon(t-s),
$$

where $\rho^{\prime}(u)=\frac{d \rho(u)}{d u}$ and $\mathcal{Y}(t)=\sum_{s \in Y} \delta(t-s)$ is the spike train of the readout neuron as a sum of $\delta$ functions. Weights are updated as $\dot{w}_{j}(t)=\eta \mathcal{R} e_{j}(t)$, with learning rate $\eta$ and reward signal $\mathcal{R}$. It is unrealistic however, to assume that reward can be delivered instantaneously at every moment in time. Therefore, $e_{j}(t)$ is low-pass filtered to provide a moving average called the synaptic eligibility trace $E_{j}(t)[7,18]$, given as:

$$
\tau_{\mathcal{R}} \dot{E}_{j}(t)=e_{j}(t)-E_{j}(t),
$$

where the time constant $\tau_{\mathcal{R}}$ is matched to the duration of the input pattern $\mathbf{X}$. In our simulations, weights were updated only at the end of each episodic presentation of $\mathbf{X}$ when reward became available, where we set the duration of each episode $T=500 \mathrm{~ms}$. 
For the A\&C model, we determined the eligibility as:

$$
\begin{aligned}
e_{j}^{\mathrm{A} \& \mathrm{C}}(t) & =\mathcal{A}(u)\left[\mathcal{Y}(t)-\rho^{\mathrm{A} \& \mathrm{C}}(u, \dot{u})\right] \sum_{s \in X_{j}} \epsilon(t-s) \quad \text { with } \\
\mathcal{A}(u) & =\frac{2}{\sigma}\left(\frac{1}{\sqrt{\pi}} \frac{\exp \left\{-\frac{[u-\vartheta]^{2}}{\sigma^{2}}\right\}}{1+\operatorname{erf}\left\{-\frac{u-\vartheta}{\sigma}\right\}}-\frac{u-\vartheta}{\sigma}\right)
\end{aligned}
$$

where we neglected terms containing higher order time derivatives of $\dot{u}$. Preliminary simulations showed that such contributions were minimal, having little overall impact on learning. For the EXP model, the eligibility is simply given as:

$$
e_{j}^{\mathrm{EXP}}(t)=\beta\left[\mathcal{Y}(t)-\rho^{\mathrm{EXP}}(u)\right] \sum_{s \in X_{j}} \epsilon(t-s) .
$$

\subsection{Learning a Target Spike Train}

We wish to teach the network to respond to a spatio-temporal spiking pattern $\mathbf{X}$ with an output spike train $Y^{\text {out }}$, matching an arbitrary target spike train $Y^{\text {ref }}$. Similar in approach to $[6,5]$, we use the van Rossum Distance (vRD) [16] to measure the dissimilarity between $Y^{\text {out }}$ and $Y^{\text {ref }}$, giving the metric $\mathcal{D}$. We arbitrarily set the coincidence time constant $\tau_{c}=15 \mathrm{~ms}$. To remove the dependence of the vRD on the number of target spikes, $\mathcal{D}$ is normalized by setting $\mathcal{D}_{N}=\mathcal{D} / \mathcal{D}_{0}$, where $\mathcal{D}_{0}$ is the vRD from just $Y^{\text {ref }} . \mathcal{D}_{N} \in[0, \infty)$ is then mapped to a reward value $\mathcal{R} \in(0,1]$ as $\mathcal{R}=\exp \left(-\alpha \mathcal{D}_{N}\right)$, where we set $\alpha=4$ such that reward becomes negligible for distances $\mathcal{D}_{N}>1$. Maximum reward $\mathcal{R}=1$ is attained when $\mathcal{D}_{N}=0$, corresponding to a perfect match between the spike trains $Y^{\text {out }}$ and $Y^{\text {ref }} \cdot \mathcal{D}_{N}$ is determined when the presentation of $\mathbf{X}$ to the network terminates. We additionally set $\mathcal{R}=0$ when no output spikes are generated, since a lack of firing activity would lead to stagnation in learning.

Rather than directly substitute $\mathcal{R}$ into the weight update rule, we implement an adaptation of the Temporal Difference (TD) error rule, originally defined in classical Reinforcement Learning [1]. Following [6], the TD error on the $n^{t h}$ episodic presentation of the input pattern is given as $\delta_{\mathcal{R}}(n)=\mathcal{R}(n)-\langle\mathcal{R}\rangle$, with the moving average of reward updated as $\langle\mathcal{R}\rangle \leftarrow 0.1 \mathcal{R}(n)+0.9\langle\mathcal{R}\rangle$. The update for the $j^{\text {th }}$ synaptic weight after the $n^{\text {th }}$ presentation of $\mathbf{X}$ then becomes:

$$
\Delta w_{j}(n)=\eta \delta_{\mathcal{R}}(n) E_{j}(T),
$$

where $E_{j}(T)$ is the $j^{t h}$ synaptic eligibility trace at the end of the $n^{\text {th }}$ episode, with time $t=T$.

\subsection{Plasticity Rules}

For learning, we implement 'additive' STDP [13], where synaptic weight changes $\Delta w_{j}$ are simply clipped if the absolute value $\left|w_{j}\right|$ moves outside of the range $\left[w_{\min }, w_{\max }\right]$. We set $w_{\min }=5 \times 10^{-3}$ and $w_{\max }=5$ as the minimum and 
maximum attainable absolute synaptic weights respectively. In all cases, plasticity takes place in both excitatory and inhibitory connections, where inhibitory connections have negative values for $w_{j}$.

To maintain a homeostatic firing rate and introduce competition between afferent connections, a simplified adaptation of the synaptic scaling rule proposed by $[19]$ is used:

$$
\Delta w_{j}^{\text {scaling }}=\gamma\left|w_{j}\right|\left[N^{r e f}-N^{\text {out }}\right],
$$

where $\gamma$ is the scaling strength, $N^{\text {ref }}$ the number of target spikes and $N^{\text {out }}$ the number of spikes generated by the readout neuron over the duration of each learning episode. Weight changes from scaling are implemented 'additively' and take place at the end of each learning episode, where we set the scaling strength $\gamma=1 \times 10^{-3}$.

\subsection{Network Setup and Learning Task}

We implemented a two-layer fully-connected feedforward network, consisting of 500 neurons in the first layer and a single readout neuron in the second layer. Either the A\&C or EXP model defined the readout neuron. The input pattern $\mathbf{X}$ consisted of an independent Poisson-distributed spike train for every input neuron, each with a mean firing rate of $6 \mathrm{~Hz}$. Synaptic weights between the first layer and readout neuron were initialized by independently selecting each value from a Gaussian distribution, with means 0.32 and 0.26 for $\mathrm{A} \& \mathrm{C}$ and EXP respectively and the standard deviation $1 / 3$ the mean. These values were selected to drive the initial firing rate of the readout neuron to $6 \mathrm{~Hz}$. The ratio of excitatory to inhibitory weights was $4: 1$.

For each learning task the network had to learn to reproduce a target spike train, with spikes selected from a uniform distribution over [50,T - 50]. For multiple-spike target trains, target spikes were separated by a minimum of $2 \tau_{c}$ to avoid confliction.

\section{Results}

We explored the capability of both the A\&C and EXP model in learning to reproduce an arbitrary target spike train in response to a fixed input pattern, where the number of target spikes ranged from 1-10. In all simulations we set the learning rate to $\eta=200$ for both A\&C and EXP readout neurons.

To characterise learning, we defined a performance measure $p$ such that desirable responses by the network gave $p=100 \%$ and $p=0$ otherwise. We considered desirable responses to occur on those episodes where every target spike could be paired to within $\Delta t=10 \mathrm{~ms}$ of an output spike, given that such values for $\Delta t$ between output and target spikes had the effect of reducing $\mathcal{D}_{N}$. We additionally set the constraint that the output spike train must contain the same number of spikes as its target, thereby disallowing spurious spiking. Since there were large fluctuations in the output with each episode, we took the performance as a moving average. The average performance was updated on each episode according 

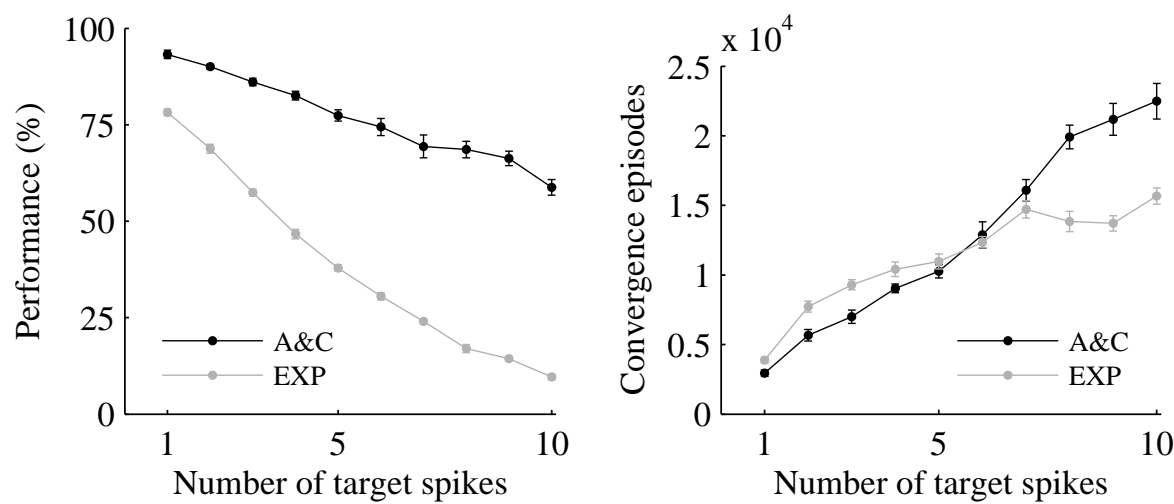

Fig. 1. Each network learning to reproduce an arbitrary N-spike target train in response to a fixed input pattern. (Left) Performance $\tilde{p}$ at convergent episode number $n_{c}$ for each network. (Right) Number of episodes to convergence $n_{c}$ in the performance $\tilde{p}$ for each network. Each point is the mean over between 10-20 independent learning tasks, where error bars show the standard error of the mean.

to $\tilde{p}(n)=(1-\lambda) \tilde{p}(n-1)+\lambda p(n)$ with $\lambda=0.004 . \tilde{p}(n)$ measured the probability of the network generating a desired response on the $n^{\text {th }}$ episode. To measure the convergence in learning, we took a similar approach as Florian [7]: convergence was considered to take place on episode number $n_{c}$ if $\tilde{p}(n)$ did not become larger than $\tilde{p}\left(n_{c}\right)$ for episode numbers between $n_{c}$ and $n_{c}+\nu$. The convergence observation period $\nu$ was set between 1000-5000 episodes, scaling with the number of target spikes to be learnt.

Fig. 1 shows the performance and convergence speed of the A\&C and EXP escape rates when learning to reproduce 1-10 target spikes. We found that A\&C consistently outperformed EXP, where the difference in $\tilde{p}$ between the two models approached 50 percentage points for 10 target spikes. A\&C maintained a good level of performance over the entire range of target spike trains we considered, with a minimum of $\tilde{p}=59 \pm 2 \%$ for 10 target spikes. By contrast, the performance of EXP deteriorated rapidly, with $\tilde{p}=9.6 \pm 0.7 \%$ for 10 target spikes. In terms of the convergence speed, A\&C converged more rapidly than EXP from between 1 and 5 target spikes, although the reverse was found for target spikes greater than 5, where the convergence time for EXP saturated. For A\&C, there was an indication of decreasing convergence rate from 8 target spikes. The decreased number of convergence episodes for EXP reflected the relatively fast attainment of poorer convergent performance.

For illustration, we show a typical spike raster for each escape rate when learning to reproduce an 8-spike target train, shown in Fig. 2. Clearly, A\&C outperformed EXP, where we found A\&C took just over 7500 episodes to attain a performance of $50 \%$, corresponding to a performance of $\sim 10 \%$ for EXP. According to Fig. 1, the number of episodes to convergence in $\tilde{p}$ for 8 target spikes were $n_{c}=(1.99 \pm 0.08) \times 10^{4}$ and $n_{c}=(1.38 \pm 0.07) \times 10^{4}$ for A\&C 

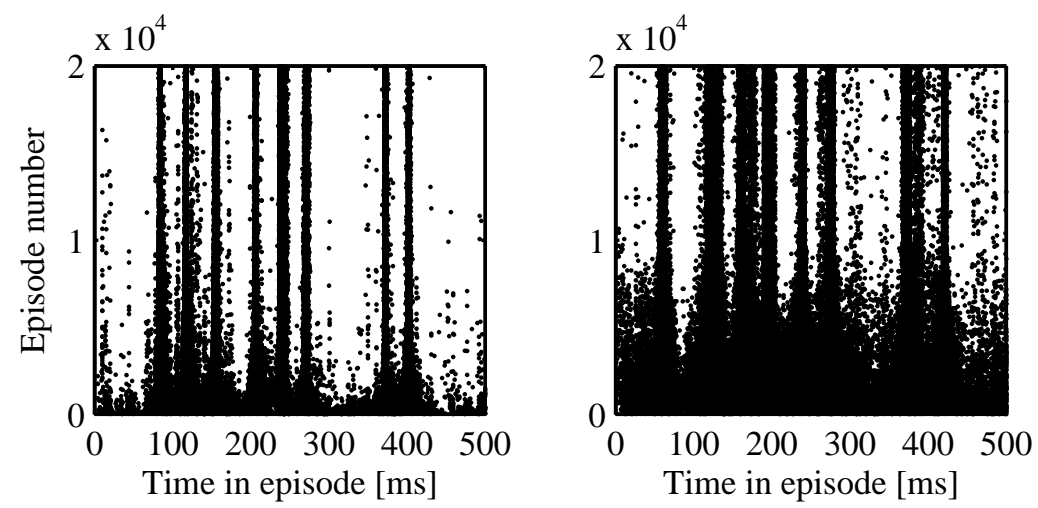

Fig. 2. Learning to reproduce an 8-spike target train in response to a fixed input pattern. Both spike rasters reflect typical network responses. (Left) A\&C model. (Right) EXP model. Note the broader spread of the EXP model around the target spike times.

and EXP respectively. In relation to Fig. 2, these values for $n_{c}$ indicated those episodes beyond which no further gains in performance were possible. Although it might appear that performance converged earlier, especially for $\mathrm{A} \& \mathrm{C}$, there existed an intermediate period of 'fine-tuning', during which spurious spiking was further reduced to allow for relatively smaller but significant gains in the performance.

\section{Discussion}

We have explored the utility of a stochastic neuron model in learning to reproduce temporally precise spiking patterns through reward modulated STDP, a process that might underpin learning in the brain. Furthermore, we have investigated two different escape rate functions to drive neuronal spiking, and compared their performance over a range of target spike trains. We found using an escape noise neuron model to be ideally suited to the task of reproducing target spike trains by reinforcement, given that a degree of background noise was essential in driving explorative spiking during learning. In terms of the escape rate model, A\&C performed consistently better than EXP for the set of parameters considered, with the difference in performance being apparent for a larger number of target spikes. We were, however, primarily motivated in applying the A\&C rather than just the EXP model given its dependence on the experimentally measurable noise amplitude parameter $\sigma$.

In our network we only included one readout neuron for simplicity. More realistically we can expect populations of neurons processing similar input patterns, where the output can be "averaged over" (for example by a leaky integrator) to produce a more deterministic response. Given that our simulations were limited to learning single input-output pattern pairs, future work utilizing such populations would likely facilitate the learning of several such pattern pairs. 
Acknowledgement. BG was fully supported by EPSRC grant EP/J500562/1.

\section{References}

1. Barto, A., Sutton, R.: Reinforcement learning: An introduction. MIT press Cambridge, MA (1998)

2. Bi, G., Poo, M.: Synaptic modifications in cultured hippocampal neurons: dependence on spike timing, synaptic strength, and postsynaptic cell type. The Journal of Neuroscience 18(24), 10464-10472 (1998)

3. Bohte, S.: The evidence for neural information processing with precise spike-times: A survey. Natural Computing 3(2), 195-206 (2004)

4. Chance, F., Abbott, L., Reyes, A.: Gain modulation from background synaptic input. Neuron 35(4), 773-782 (2002)

5. El-Laithy, K., Bogdan, M.: A reinforcement learning framework for spiking networks with dynamic synapses. Computational Intelligence and Neuroscience 2011, $4(2011)$

6. Farries, M., Fairhall, A.: Reinforcement learning with modulated spike timing dependent synaptic plasticity. Journal of Neurophysiology 98(6), 3648-3665 (2007)

7. Florian, R.: Reinforcement learning through modulation of spike-timing-dependent synaptic plasticity. Neural Computation 19(6), 1468-1502 (2007)

8. Frémaux, N., Sprekeler, H., Gerstner, W.: Functional requirements for rewardmodulated spike-timing-dependent plasticity. The Journal of Neuroscience 30(40), 13326-13337 (2010)

9. Gerstner, W., Kistler, W.: Spiking neuron models: Single neurons, populations, plasticity. Cambridge University Press, Cambridge (2002)

10. Grüning, A., Sporea, I.: Supervised learning of logical operations in layered spiking neural networks with spike train encoding. Neural Processing Letters 36(2), 117$134(2012)$

11. Izhikevich, E.: Solving the distal reward problem through linkage of stdp and dopamine signaling. Cerebral Cortex 17(10), 2443-2452 (2007)

12. Legenstein, R., Pecevski, D., Maass, W.: A learning theory for reward-modulated spike-timing-dependent plasticity with application to biofeedback. PLoS Computational Biology 4(10), e1000180 (2008)

13. Morrison, A., Diesmann, M., Gerstner, W.: Phenomenological models of synaptic plasticity based on spike timing. Biological Cybernetics 98(6), 459-478 (2008)

14. Pfister, J., Toyoizumi, T., Barber, D., Gerstner, W.: Optimal spike-timingdependent plasticity for precise action potential firing in supervised learning. Neural Computation 18(6), 1318-1348 (2006)

15. Ponulak, F., Kasinski, A.: Supervised learning in spiking neural networks with resume: Sequence learning, classification, and spike shifting. Neural Computation 22(2), 467-510 (2010)

16. Rossum, M.: A novel spike distance. Neural Computation 13(4), 751-763 (2001)

17. Sporea, I., Grüning, A.: Supervised learning in multilayer spiking neural networks. Neural Computation 25(2), 473-509 (2013)

18. Urbanczik, R., Senn, W.: Reinforcement learning in populations of spiking neurons. Nature Neuroscience 12(3), 250-252 (2009)

19. Van Rossum, M., Bi, G., Turrigiano, G.: Stable hebbian learning from spike timingdependent plasticity. The Journal of Neuroscience 20(23), 8812-8821 (2000) 